\title{
COVID-19 geoviz for spatio-temporal structures detection
}

\author{
Jacques Gautier ${ }^{\text {a*}}$, Maria-Jesus Lobo ${ }^{a}$, Benjamin Fau ${ }^{\text {a }}$, Armand Drugeon ${ }^{\text {a }}$, Sidonie \\ Christophe $^{\text {a }}$, Guillaume Touya ${ }^{\text {a }}$
}

a LASTIG, Univ Gustave Eiffel, ENSG, IGN, F-94160 Saint-Mande, France

* Corresponding Author

\begin{abstract}
The spread of COVID-19 has motivated a wide interest in visualization tools to represent the pandemic's spatio-temporal evolution. This tools usually rely on dashboard environments which depict COVID-19 data as temporal series related to different indicators (number of cases, deaths) calculated for several spatial entities at different scales (countries or regions). In these tools, diagrams (line charts or histograms) display the temporal component of data, and 2D cartographic representations display the spatial distribution of data at one moment in time. In this paper, we aim at proposing novel visualization designs in order to help medical experts to detect spatio-temporal structures such as clusters of cases and spatial axes of propagation of the epidemic, through a visual analysis of detailed COVID-19 event data. In this context, we investigate and revisit two visualizations, one based on the Growth Ring Map technique and the other based on the space-time cube applied on a spatial hexagonal grid. We assess the potential of these visualizations for the visual analysis of COVID-19 event data, through two proofs of concept using synthetic cases data and web-based prototypes. The Grow Ring Map visualization appears to facilitate the identification of clusters and propagation axes in the cases distribution, while the space-time cube appears to be suited for the identification of local temporal trends.
\end{abstract}

Keywords: spatio-temporal visualization; epidemic visualization; spatio-temporal structures; exploratory analysis; 3D visualization

\section{Introduction}

The spread of COVID-19 has quickly motivated creating and sharing diverse visualizations to represent the spatiotemporal evolution of the pandemic for various purposes of communication, analysis and decision-making (Müller and Louwsma, 2021). A lot of these visualizations propose to explore the distribution of COVID-19-related events, such as cases' occurrences and deaths along time and space, into multi-view "dashboard" environments where the temporal and spatial components of the data are visualized in dedicated synchronized windows (Kraak and Ormeling, 2011), such as the John Hopkins dashboard ${ }^{1}$ or the World Health Organization dashboard tracker ${ }^{2}$. In most cases, the represented data are temporal series related to indicators (number of new or accumulated cases, deaths, basic reproduction number) calculated for different spatial entities such as countries, administrative regions or cities.

The temporal component of COVID-19 data is visualized in these environments through one (or several) temporal diagram(s): representation of the linear evolution of cases at a global scale through a linear curve or a histogram ${ }^{3}$; representation of the linear evolution of the number of cases at different spatial locations through a collection of temporal diagrams ${ }^{4}$; branching time representations ${ }^{5}$, highlighting decisive events corresponding to the birth of new versions of the virus. The spatial component of the data is mainly tackled by simple 2D maps, representing the data related to the corresponding spatial entities for a selected timestep

\footnotetext{
${ }^{1}$ https://coronavirus.jhu.edu/map.html

${ }^{2}$ https://covid19.who.int/

${ }^{3}$ https://hgis.uw.edu/virus/

${ }^{4}$ https://boogheta.github.io/coronavirus-countries/

${ }^{5}$ https://nextstrain.org/ncov/global
}

or temporal range ${ }^{6}$, such as a representation of the number of cases by country, occurring at or until a certain date, selected by the user using a dynamic temporal representation. In order to transcribe the dynamics of the phenomena, some environments propose animation techniques ${ }^{7}$, allowing the user to successively visualize the spatial component of data for different timesteps.

The understanding of the dynamics of spatio-temporal phenomena, such as the COVID-19 pandemic, would benefit from visualization environments allowing the visual exploration of the spatio-temporal distribution of event data, describing the date and place of each new event such as a new COVID-19 case. Spatial or spatio-temporal analysis has been often used to find clusters of epidemic cases (concentration of cases in space and time) (Delmelle et al., 2014, Kirby et al., 2017), in particular when a precise location of cases is available (Piarroux, 2019). Such analyses are already used to study the propagation of the COVID-19 pandemic (Desjardins et al., 2020, Liu et al., 2020, Purwanto et al., 2021). The structures highlighted with these analysis, such as clusters, axes of propagation and local trends, can then be depicted graphically through visualizations.

Some dashboard exploratory analysis environments such as Persee (Swedberg and Peuquet, 2017), Timewave (Li, 2010) or GrAPHiST (Gautier et al., 2020) propose to visualize the entire spatio-temporal distribution of event data at once, through different approaches such as small multiples and/or representing the temporal component of data by visual variables in a map, in order to identify structures with a visual analysis. Specific designs allow the user to identify global structures from an overall visualization of

\footnotetext{
${ }^{6}$ https://www.arcorama.fr/2020/03/un-tableau-de-bord-arcgistoujours-plus.html

${ }^{7}$ https://ourworldindata.org/coronavirus
} 
the spatio-temporal distribution of event data. Interaction functionalities allow then to navigate in the data in order to perform a more precise analysis of the observed structures.

In this paper, we aim at proposing novel visualization designs of COVID-19 event data inspired by these environments, representing each case occurrence, dedicated to the expectations of medical experts in order to help them better understand the pandemic's spreading through visual analysis. Medical experts are especially interested in detecting spatio-temporal structures such as local clusters, propagation axes and local trends. Identifying such structures would help them better interpret and understand the virus transmission mechanisms. To achieve this goal, we propose to integrate both the spatial and temporal components of the COVID-19 cases into the same graphic representation, as to help users relate spatial and temporal structures.

In order to perform a visual identification of COVID-19 clusters, we experiment two strategies to visually represent the spatio-temporal distribution of cases in spatial representations. The remaining of the paper is organized as follows. First, we review existing work related to spatiotemporal visualizations. Second, we detail the design of the two approaches considered, the first one based on the Growth Ring Map visualization and the second one based on the space-time cube. Finally, we discuss the potential of both visualizations through the exploration of synthetic COVID-19 data, and highlight their current limitations.

\section{Related Work}

\subsection{The role of map design for the visual identification of structures in event data distribution}

Visualizing spatio-temporal data may be addressed by the following approaches (Gautier et al., 2020): (1) the representation of data temporal components on graphic objects into a spatial representation, through visual variables such as shape or color value/hue (Saint-Marc et al., 2014); (2) the use of temporal diagrams integrated into a map and related to spatial features (Huang et al., 2008); (3) the use of small multiples, a collection of spatial representations related to different timesteps or a collection of diagrams related to different spatial locations (Tufte, 1983, Davoine et al., 2015); (4) the use of the vertical dimension of 3D representations to represent the time through the space-time cube proposition (Gatalsky et al., 2004); and (5) the use of animation (Harrower et al., 2008). Furthermore, the choice of the time structures to represent can affect the choice of graphic designs in these different approaches. Considering the use of visual variables, the evolution of color value can represent the linear evolution of time, but the use of the evolution of color hue can be more suited to represent a cyclical evolution of time (Gautier et al., 2020). Furthermore, considering the use of integrated temporal diagrams in maps, the use of circular timewheel-based diagrams instead of timeline-based diagrams can be more suited to represent cyclic phenomena (Auer et al., 2011).

When visualizing a massive amount of event data, all of these approaches might suffer from visual clutter. Previous works propose map designs based on a spatial aggregation of event data and the graphic representation of these aggregates, to alleviate this problem. (Delort, 2010, Meier, 2016). This aggregation can be performed according to the spatial distance between data (two events are aggregated together if the distance between their locations or the graphic distance between their representations on the map is below a specific threshold), or according to a spatial grid (all events covered by a grid's cell are aggregated together). Furthermore, specific designs applied to the representation of the events aggregates can also be used to enhance the visual identification of spatio-temporal structures such as clusters or local trends in events' distribution (Seebacher et al., 2018, Choi et al., 2018, Gautier et al., 2020).

These approaches could be used to co-visualize the spatial distribution of event data, enabling the identification of structures. For example, some of them have already been used specifically for COVID-19 data, such as glyph-based representations (Beecham et al., 2021, Lan et al., 2021) and 3D representations (Lan et al., 2021). However, temporal animation is not considered as the best option for the visual exploration of spatio-temporal data (Harrower et al., 2008), and the use of small multiples or integrated diagrams in $2 \mathrm{D}$ representations can be problematic in case of a high number of timesteps or spatial locations to covisualize (Davoine et al., 2015). Therefore, we review more in detail the use of space-time cubes and time representation by visual variables to visualize the distribution of COVID-19-related event data in space and time, and to identify clusters, propagation axes, and local trends.

\subsection{Representation of time by visual variables}

Different semiologic rules have been edicted to project on graphic objects, with the visual variables defined by Jacques Bertin (Bertin, 1973), different temporalities such as the moment of appearance, the duration or the frequency of events (Dukaczewski, 2005, Cauvin et al., 2010) in 2D or $3 \mathrm{D}$ representations. The use of dynamic variables using animation, such as the use of blinking features, to represent temporalities has also been described (Green, 1998, MacEachren, 2004). Of course, the application of such rules is still ambiguous and depends on how the temporal component of a data is interpreted as a variable (SaintMarc et al., 2014): the moment of appearance of events can be used to distinguish events occurring during different periods (and be treated as a qualitative variable represented by the color hue), or to describe an order of appearance between events (and be treated as a ordinal variable represented by the color value).

The Growth Ring Map proposition explores the possibility to enhance the visual identification of structures in the spatio-temporal distribution of events, through specific designs applied to the graphic representation of events aggregates, and the use of visual variables to represent the temporal component of data (Bak et al., 2009, Andrienko et al., 2011, Aigner et al., 2011). This proposition uses both color hue and value to represent event data according to their cyclic and linear moment of appearance: events appearing during the same season are represented with the same hue, events appearing the same year with the same value. Each event is represented by a pixel bearing the corresponding color, spatially closed events forming aggregates of pixels. In each aggregate, the pixels representing events appearing during the same season are grouped together into concentrated rings of the same color hue. Then, inside each colored ring, concentric sub-rings bearing different values of the color represent events appearing during the same year. Such representation allows identifying 


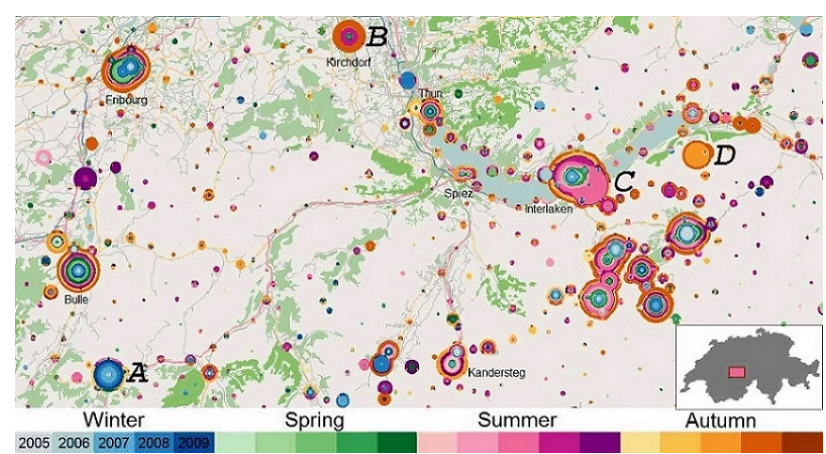

Figure 1. Representation of the spatio-temporal distribution of event data through a Growth Ring Map visualization (Bak et al., 2009, Andrienko et al., 2011, Aigner et al., 2011)

structures like spatio-temporal clusters: concentrations of events appearing at the same place each year during the same season (Fig.1-A), or at the same place during one season of one specific year (Fig.1-D).

\subsection{D representations as Space-Time Cube}

The space-time cube (STC) is a 3D visualization technique that maps geographical positions in the $\mathrm{x}$ and $\mathrm{y}$ coordinates and time vertically (Hägerstraand et al., 1970). The spacetime cube has been used to visualize trajectories (Kraak, 2008, Gonçalves et al., 2016), discrete events such as earthquakes (Gatalsky et al., 2004), crime events and disease data (Kraak, 2008, , n.d.), and in particular to visualize COVID data (Lan et al., 2021). Because plotting many events might result in occlusion, some approaches explore aggregating them spatially or temporally and using an alternative representation such as density-based representations (Demšar et al., 2015) or helix representations (League and Kennelly, 2019). Current techniques rely on interactivity to enable users to manipulate the view, for example to change the point of view or filter the data (Kapler and Wright, 2005, Amini et al., 2014).

Results of empirical studies comparing 2D and 3D representations for spatio-temporal data suggest that STC might be more efficient for overview tasks (Gatalsky et al., 2004) while $2 \mathrm{D}$ views perform better for simple and location tasks. A user study comparing a 2D view, a STC and a combination of both for trajectory visualization has been fulfilled, suggesting that is better using a combination of both views, rather than using them individually (Gonçalves et al., 2016).

In summary, multiple approaches exist to depict spatiotemporal structures in event data. Given the evidence that $2 \mathrm{D}$ views and $3 \mathrm{D}$ views might be complementary, we propose using, in parallel, a $2 \mathrm{D}$ view using visual variables to represent the temporal component of data and a $3 \mathrm{D}$ view using the third dimension to depict time to represent disease events. In order to reduce visual cluttering, we use aggregation methods for both views and we explore various designs to enhance the visual identification of structures in events' spatio-temporal distribution.

\section{Complementary event data Visualization designs}

We propose two complementary visualizations aiming to ease the visual identification of structures in the spatiotemporal distribution of COVID-19 individual case data.
We consider their design specificities, and how they can depict and facilitate the identification of structures. The main goals of these visualizations are the following:

- Enabling an interactive exploration of the data;

- Presenting both an overview and a detailed view of the cases distribution;

- Emphasizing spatio-temporal structures such as clusters, propagation axes and local temporal trends.

First, a 2D view, based on the Growth Ring Map, depicting spatio-temporal evolutions through the use of visual variables representing time on graphic objects and specific map designs allowing the visual identification of structures in the distribution of event data. Second, a 3D view based on a space-time cube, and providing a global view of the data by using the third dimension to depict time. Because both visualizations suffer from clutter when displaying many events, we propose different aggregation methods for each visualization.

\subsection{Data}

Because of privacy issues, we did not have access to the data for individual cases. Thus, as a case study for our proof of concept, we generated synthetic data. We acknowledge there are more accurate simulations of epidemics' spreading. We tried yet to reproduce their basic dynamics to assess our visualizations' potential. In order to generate structures similar to the ones usually present in epidemic's data, we set the following criteria to generate event data, where each event corresponds to a case of the disease:

- At the start of the epidemic, the position of each case is random.

- Each case remains active for a fixed number of days. Each active case can generate new cases, with a higher probability in its neighborhood than elsewhere.

- Each case has a fixed and constant probability of generating new cases.

Following our criteria, each case is described by its spatial coordinates, and its dates of infection, end of incubation period, and end of contagious period. We consider that a case is active between the end of its incubation period and the end of its contagious period. We consider than a case is spatially static and doesn't change of location.

The criteria we chose to determine the creation process of our data are inspired from the information given by the French ministry of Health ${ }^{8}$. The incubation period's length is mainly between 3 and 5 days but can reach 14 days; the contagious period is mainly around 8 days after the first symptoms. We retain the main values for each period's length and consider that the incubation period's length is between 3 to 5 days, and contagious period's length is between 7 to 9 days. We considered that during each day of its active period, a case has 17.5 percent of chance to generate one new case. This percentage has been defined empirically, to obtain around 10000 cases from our initial set of cases after 100 days. Combined with an average contagious period of 8 days, this percentage corresponds

\footnotetext{
${ }^{8}$ https://www.gouvernement.fr/info-coronavirus/comprendre-lacovid-19
} 


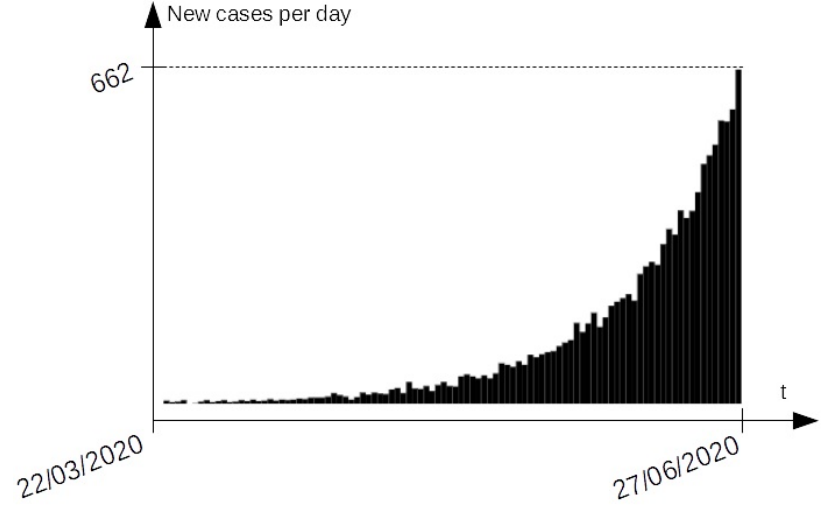

Figure 2. Temporal distribution of synthetic data

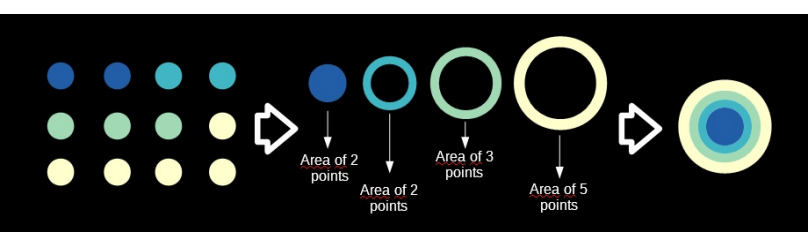

Figure 3. Construction of a glyph representing an aggregate of points

to a constant basic reproduction number (R0) of 1.4 (each infected case generating an average number of 1.4 new cases). As a comparison, such a value for the COVID-19's R0 was reached in France during the second wave's peak, the $13 / 10 / 2020^{9}$. Thus, we can consider that our dataset shows a realistic spread of the disease over time.

For the final dataset, we set the generation to start with 20 events, occurring through three days between the 19/03/2020 and the 22/03/2020 in Paris. Each new case has 20 percent of chance to be inside a circular area of 500 meters around the case of origin, 30 percent inside an area of 1000 meters, 30 percent inside an area of 2500 meters, and 20 percent inside an area of 5000 meters. These percentages have been defined empirically to generate visible spatiotemporal structures in the data. For each new case, the duration of the incubation and contagious periods is randomly chosen respectively between 3,4 and 5 days, and 7, 8 and 9 days. For the first 100 days after the 19/03/2020, we obtain a dataset of 11853 cases occurrences covering the region of Paris between the 19/03/2020 and the 27/06/2020 (Fig.2).

\subsection{Growth Ring Map Visualization}

The first visualization is based on the Growth Ring Map proposition (Bak et al., 2009, Andrienko et al., 2011). As in the previous proposition, we use the color to represent the temporal moment of appearance of the COVID-19 cases. As we said before, we focus our proposition on the visualization of linear temporal structures in our data. Color value is then used to represent the linear order of appearance of our cases. We determine a color scale covering the temporal extent represented on the map, each color representing a regular, or irregular, sub-interval of time. As the color hue is not used to represent any other component of the data, we can use the evolution of hue to enhance the distinction of the different represented temporal inter-

\footnotetext{
${ }^{9} \mathrm{https} / / / \mathrm{www} . g o u v e r n e m e n t . f r / i n f o-c o r o n a v i r u s / c a r t e-e t-d o n n e e s$
}

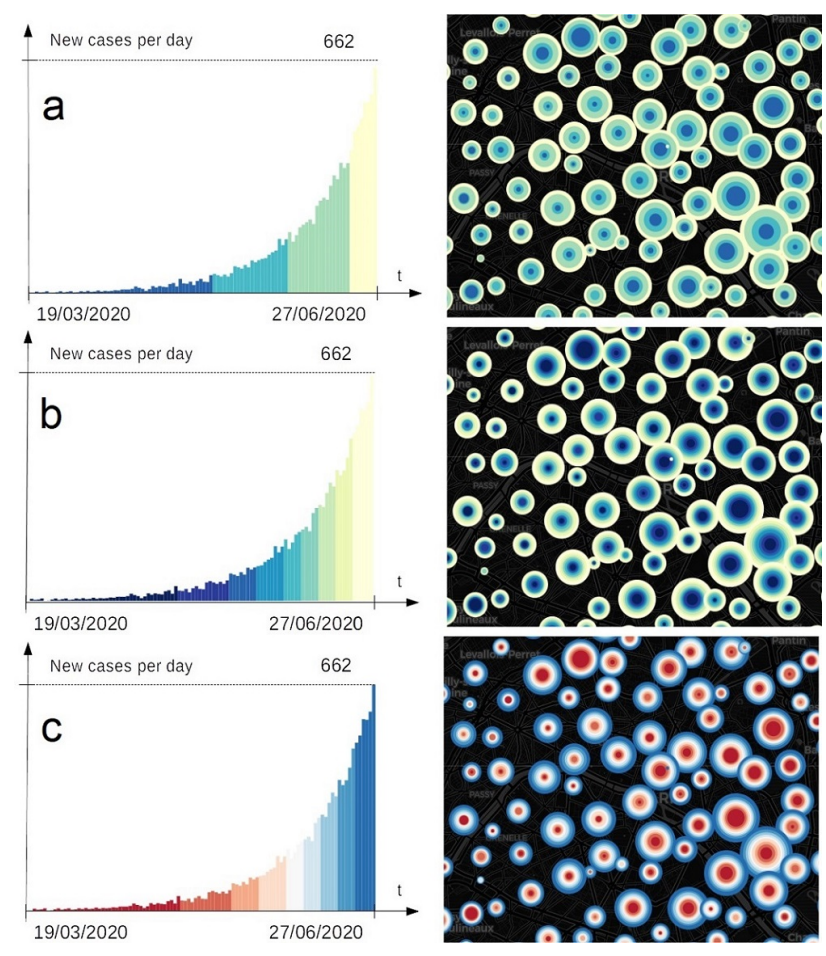

Figure 4. Visualization of the spatio-temporal distribution of the COVID-19 synthetic data, using a) a multi-hue sequential color scale of 4 colors, b) a multi-hue sequential color scale of 9 colors, c) a diverging color scale of 9 colors

vals, though the use of multi-hue sequential color-scales (Brewer, 1994).

We represent the COVID-19 cases occurrences (referred as "events" in the remaining of the paper) by a cloud of punctual features. In order to reduce visual cluttering and highlight spatial concentrations of events, the spatially close ones are aggregated and represented by a unique punctual feature whose area represents the number of aggregated events. A unique event is then represented by a point bearing a color corresponding to the temporal interval during which the event occurs. An aggregate of events, instead, is represented by a punctual circular glyph composed of concentric rings of different color, each representing the proportion of aggregated events appearing during the corresponding temporal interval represented in the color scale (Fig.3). For each ring, the proportion of the corresponding events in the aggregate is represented by the area of the glyph covered by the ring surface. The different concentric rings are disposed according to the order of appearance of the corresponding intervals, with the older intervals represented by the inner rings and the latest intervals represented by the outer rings (this order can also be reversed).

The color scales we use can present different granularities. Scales with a low number of colors are easier to use (Fig.4-a), the distinction between colors being easier than with color scales using more colors (Fig.4-b). However, the represented temporal intervals being wider with a low number of colors, these scales would not be the best suited for the identification of clusters with a smaller temporal extent than the ones represented by each color. We propose to use diverging color scales (Fig.4-c), in order to represent smaller temporal intervals with each color, and still being able to distinguish, inside an aggregate of points, at least 

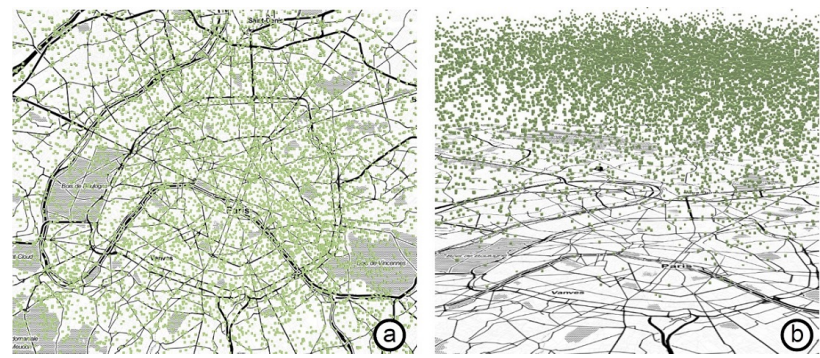

Figure 5. Spatio-temporal 3D visualization representing each event as a point: (a) horizontal view (b) vertical view

events appearing during the newest, latest, and intermediate temporal intervals represented by the color scale. To enhance the perception of the different colors in the map, we use a basemap using dark grey and black colors, which would be less confusing with the color scale.

Users are able to navigate in the representation through pan and zoom functionalities, and to dynamically change the represented temporal extent, the color scale and the corresponding temporal intervals, the range of the spatial aggregation and the general size of the glyphs. The events' aggregation is dynamic and depends on the zoom level chosen by the user. Two aggregated events can be represented as separate at a lower scale.

We implemented our proposition in a Leaflet-based map, using the markerclustergroup ${ }^{10}$ plugin for the aggregation of spatially closed events. The glyphs representing each aggregate of events are dynamically created in SVG.

\subsection{Space-time cube}

The second visualization we chose to explore is based on the space-time cube. As in existing applications (Gatalsky et al., 2004), we represent time vertically. Each event is represented with a cubic punctual feature, and thus clusters can be identified by searching for points that are close to each other. However, this results in a cluttered view, as depicted in Figure 5. To partially alleviate this problem, we enable users to filter the visible events by restricting the temporal interval. The visualization also relies on user interaction to enable users to change the point of view, and explore the data.

In order to reduce visual clutter, we aggregate individual events spatially and temporally. First, we spatially aggregate the events according to an hexagonal grid. Hexagons have the advantage of being more visually appealing and provide a more accurate representation than square grids (Carr et al., 1992). The number of hexagons in the grid changes interactively, as the user zooms in the visualization. This enables users to have an overview, and zoom in to get a more detailed view of each region. Furthermore, we aggregate events temporally by grouping them according to regular day intervals. Thus, each aggregate corresponds to one spatial hexagonal cell and a regular temporal interval, and is depicted as a 3D volume, whose horizontal area is proportional to the number of aggregated cases and the height represents the duration of the temporal interval. This results in a series of stacked hexagons for each region. We represent the interval length through the $3 \mathrm{D}$

\footnotetext{
${ }^{10}$ https://github.com/Leaflet/Leaflet.markercluster
}

hexagon height. Finally, to facilitate the comparison between 3D hexagons, we also use a color scale to encode the number of cases for each volume.

Users are able to freely navigate in the visualization using common 3D navigation controls: zooming, panning and rotating. Furthermore, they can select an hexagon to display its precise information (number of cases and time interval), and to highlight all the hexagons corresponding to the selected region. We defined three different aggregate representations: going from an hexagon radius of 200 meters to 50 meters, and from a temporal aggregation of 4 days to 1 . We defined these values and the corresponding zoom levels empirically, in order to allow going from an overview of the data to a detailed view. While zooming in, the users goes automatically from one aggregate level to the next one, the last level being a point cloud representation of every event. The three aggregation levels are depicted in Figure 6. Furthermore, users can also adjust the temporal scale.

The 3D visualization is implemented using Three.js, d3.js and OpenLayers.

\section{Results and Discussion}

We designed two visualizations to facilitate spatio-temporal structures detection through visual analysis of the distribution of spatio-temporal event data such as COVID-19 cases datasets. To assess the potential uses of the two proposed designs, we analyzed the spatio-temporal distribution of our synthesized data.

\subsection{D representation advantages: identification of clus- ters and propagation axes}

We analyzed the data through the Growth Ring Map visualization, first on their entire temporal extent (19/03 to 27/06) using a blue-red diverging color scale representing 11 regular temporal intervals (Fig.7-a and Fig.7-c, top), then on a reduced period (19/03 to 16/05) using a bluered diverging color scale representing 9 regular temporal intervals (Fig.7-b and Fig.7-c, bottom). On both these visualizations, the blue color represents the latest events.

The analysis of the data on their entire temporal extent allowed us to identify the areas where the oldest events are concentrated, by observing the location of glyphs presenting a high proportion of dark red color (white frames on Fig.7-a), and the areas where the newest events are concentrated, by observing the location of glyphs presenting a high proportion of dark blue color (green frames on Fig.7a). The identification of concentration of oldest and newest events is also possible through the analysis of the data on the reduced period (19/03 to 16/05) (white and green frames in Fig.7-b). Also, in both analyses, it is possible to locate concentrations of events occurring during intermediate temporal intervals, by observing the location of glyphs presenting a high proportion of white, or light red/blue colors (purple frames in Fig.7-a and Fig.7-b). The corresponding clusters can be identified especially through the analysis on the reduced temporal extent, because the events corresponding to these intervals are less outnumbered by the newest events (Fig.7-c, bottom). The use of a broadcast aggregation of the events allows the analysis of structures at different spatial scales, enabling for instance to make a first overview analysis of the data distribution to identify 

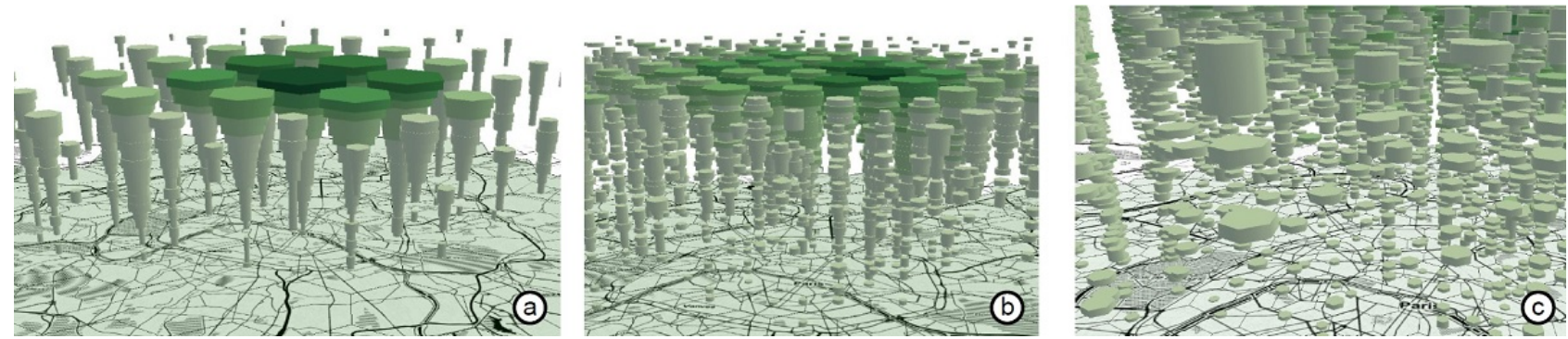

Figure 6. The three used levels of aggregation (a) Hexagons of 100m radius, grouped by 5 days, (b) hexagons of $50 \mathrm{~m}$ radius, grouped by 2 days, (c) hexagons of $25 \mathrm{~m}$ radius, by individual days

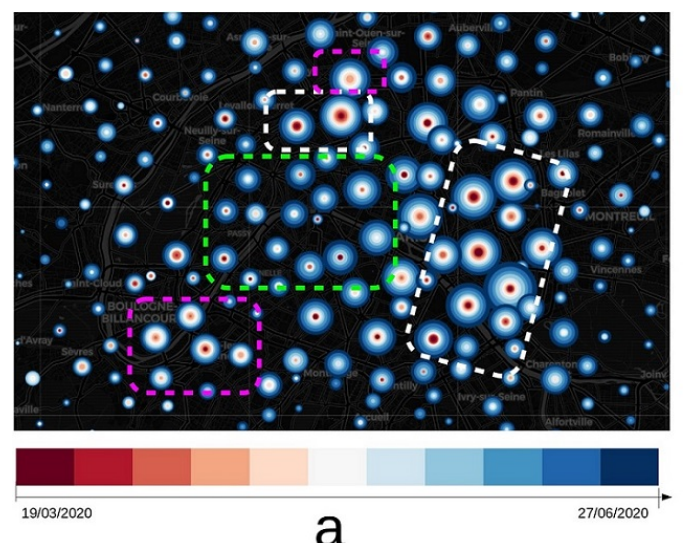

a
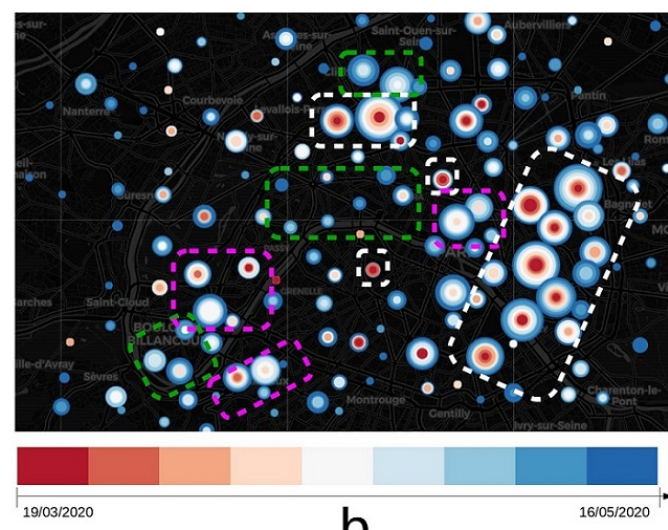

b

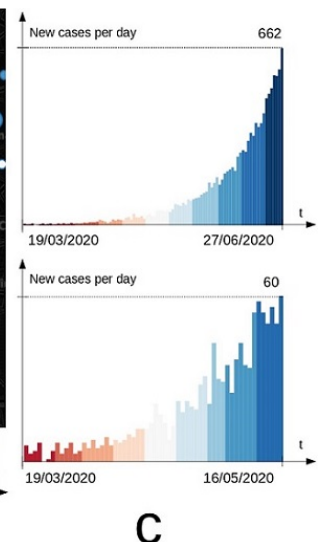

C

Figure 7. Identification of clusters in the Growth Ring Map visualization

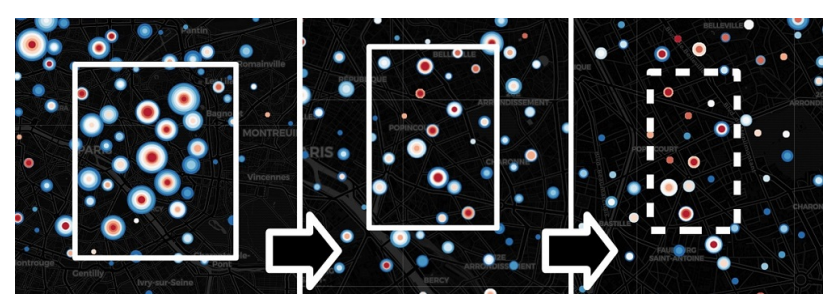

Figure 8. Analysis of clusters at different spatial scales

structures such as clusters (Fig.8, left), and then to perform successive zooms into the map (Fig.8, center and right), in order to define more precisely the spatial shape of these structures at a larger scale. Interaction functions giving the possibility to dynamically change the visualized temporal extent and the used color scale allows then defining more precisely the temporal shape of the observed structures.

For both these analyses, this representation allows the identification of spatio-temporal clusters in the distribution of events. Identifying the location of clusters corresponding to successive intervals allows then to interpret the axes of the overall propagation of cases: for instance, in the bottom-half of Fig.7-b, we observe the epidemic' spread along an east-west direction, by observing the location of clusters related to old (east), intermediate (west) and recent (far-west) intervals.

\subsection{D Representation advantages: identification of local trends}

Identifying spatio-temporal clusters using the 3D visualization might be more difficult than identifying them with the Growth Ring Map representation, because users have to compare both regions across the $\mathrm{x}$ and $\mathrm{y}$ axis, and $3 \mathrm{D}$ volumes vertically along the $\mathrm{z}$ axis, in order to look for clusters both spatially and temporally. Furthermore, comparing areas in 3D is perceptually more difficult that comparing areas in 2D because of occlusion and depth perception (Munzner, 2014). In addition, in the higher zoom levels, the aggregation into hexagons might make it difficult to find clusters that are inside a cell. Finally, the temporal and spatial aggregation might separate clusters that overlap different time and space aggregation intervals. However, when looking for temporal trends in specific regions, we might hypothesize that the $3 \mathrm{D}$ representation will better support users. To analyze the temporal evolution of a specific region, the Growth Ring Map representation requires comparing colors and arc areas, while the $3 \mathrm{D}$ representation requires comparing aligned areas. Furthermore, the hexagonal aggregation enables users to focus on a specific region, while the Growth Ring Map automatically cluster points close to each other.

For example, when looking at the whole visualization at the biggest aggregation level, users might see the regions that present the biggest number of cases, such as the Paris region (Fig.6-a). By changing the point of view (Fig.9a), users can identify the regions where the cases started early (hexagons in the smallest $\mathrm{z}$ coordinates, blue frame in Fig.9-a), or later (hexagons starting at a higher position, red frame in Fig.9-a). We see that the locations of these regions correspond to the clusters identified using the Growth Ring Map visualization. Continuing with the analysis, the user can select one specific hexagon to highlight it and its corresponding region. This reduces the occlusion problem and enables the user to get more details on the temporal evolution of that specific region. For instance, if he selects one region in the east of Paris (Fig.9-b-c), he 

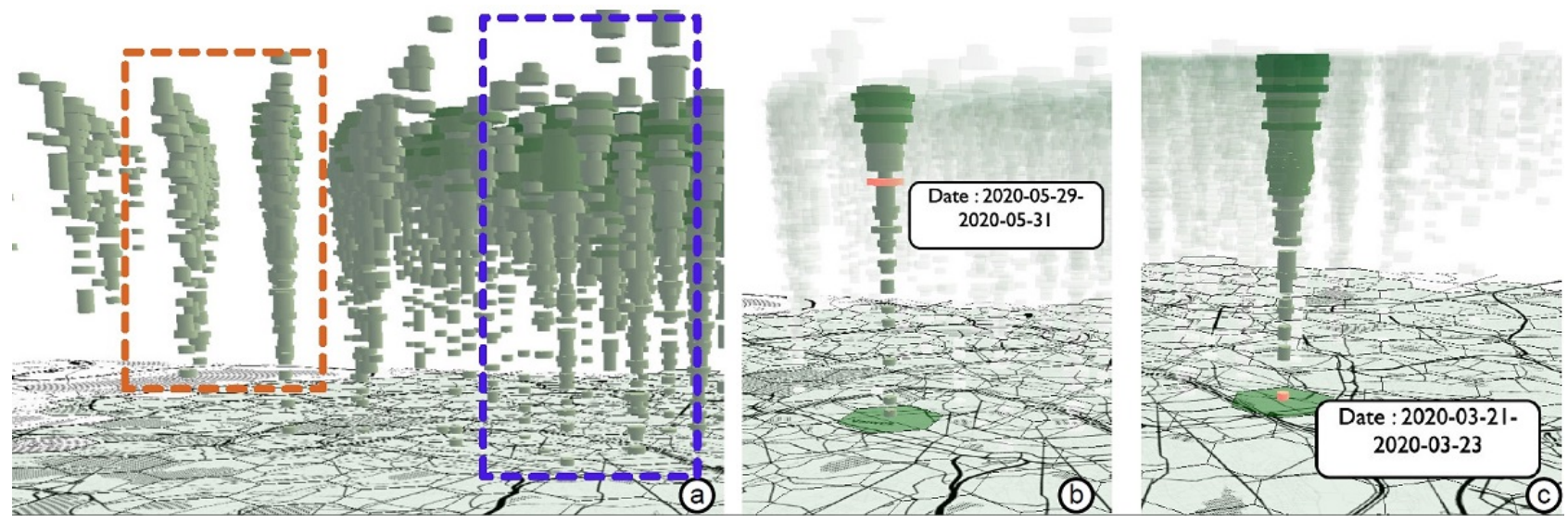

Figure 9. Exploration of the data through the 3D visualization (a) global view, regions where the propagation starts earlier (blue) and later (red), (b) highlight of a region where the propagation started in April, (c) highlight of a region where the evolution started at the beginning of the period but that only increased rapidly after may.

will see that although the cases started early and globally increased in the studied period, the daily number of cases remained stable for approximately two thirds of the time, and increased rapidly afterwards.

\subsection{Limitations}

\subsubsection{Data}

We synthesized our COVID-19 data because we did not have access to the location of individual cases for privacy issues. We acknowledge that there are methodologies to produce more accurate epidemic's simulations. For example, we do not take into account the application of public health policies or the evolution of meteorologic conditions. Furthermore, we chose to focus our proposition on the visualization of linear spatio-temporal structures in event data and we do not consider weekly or seasonal cycles. Even if this dataset is not highly realistic, we consider that it is sufficient to present the potential uses of our visualizations to identify spatio-temporal structures in real data. Further tests, with more accurate data and simulation parameters would be interesting to assess more generally the visualizations.

\subsubsection{Evaluation}

Our visualization propositions still need to be submitted to a proper evaluation, in a more controlled environment. However, the analyses we made through the visualization environments we developed already open perspectives for our future work, and we think that the presentation of our first results provide us with multiple improvement possibilities to enhance our propositions.

\subsubsection{Design choices}

For both visualizations, we chose visual encodings according to previous studies and internal validation. However, there are multiple alternatives for each visualization, regarding aggregation methods, interaction techniques and visual encodings. For example, we might use different aggregation methods, that focus on reducing visual cluttering rather than grouping close objects. Further studies, including more alternative designs, and evaluations with users would help us gain insights on which designs are better for different tasks.

\section{Conclusion}

We proposed two visualizations designed to facilitate finding spatio-temporal structures such as clusters in detailed COVID-19 data. The first one, based on the Growth Ring Map proposition, aggregates spatially closed events and uses color and specific map designs to depict the temporal composition of each aggregate of events. The events aggregation is dynamic and depends on the map's scale, enabling different levels of analysis. The second one is based on the space-time cube, and uses a 3D representation where the time is represented vertically and events are aggregated spatially through an hexagonal grid. The grid resolution varies according to the zoom level to enable going from an overview to a detailed view. We analyzed synthesized data to assess the potential of the proposed visualizations, and found that the Growth Ring Map seems to be better for detecting clusters and propagation axes, while the space-time cube seems to be more appropriate for studying regional evolution. Our approach is yet limited by the synthesized data, our design choices, and the lack of user evaluation. As future work, we plan to test other design alternatives through user studies to better assess the efficacy of the proposed visualizations. Another perspective would be to explore the possible combination of these two representations for the exploratory analysis of event data, or their combination with spatial analysis approaches performing data pre-processing in order to identify clusters, which would be explicitly visualized as spatio-temporal structures in the graphic representation.

\section{References}

Aigner, W., Miksch, S., Schumann, H. and Tominski, C., 2011. Visualization of time-oriented data. Springer Science \& Business Media.

Amini, F., Rufiange, S., Hossain, Z., Ventura, Q., Irani, P. and McGuffin, M. J., 2014. The impact of interactivity on comprehending $2 \mathrm{~d}$ and $3 \mathrm{~d}$ visualizations of movement data. IEEE transactions on visualization and computer graphics 21(1), pp. 122-135.

Andrienko, G., Andrienko, N., Bak, P., Keim, D., Kisilevich, S. and Wrobel, S., 2011. A conceptual framework and taxonomy of techniques for analyzing movement. Journal of Visual Languages \& Computing 22(3), pp. 213-232. 
Auer, T., MacEachren, A. M., McCabe, C., Pezanowski, S. and Stryker, M., 2011. Herbariaviz: A web-based clientserver interface for mapping and exploring flora observation data. Ecological Informatics 6(2), pp. 93-110.

Bak, P., Mansmann, F., Janetzko, H. and Keim, D., 2009. Spatiotemporal analysis of sensor logs using growth ring maps. IEEE transactions on visualization and computer graphics.

Beecham, R., Dykes, J., Hama, L. and Lomax, N., 2021. On the use of 'glyphmaps' for analysing the scale and temporal spread of covid-19 reported cases. ISPRS International Journal of Geo-Information 10(4), pp. 213.

Bertin, J., 1973. Sémiologie graphique. Flammarion, Paris.

Brewer, C. A., 1994. Color use guidelines for mapping. Visualization in modern cartography 1994, pp. 123-148.

Carr, D. B., Olsen, A. R. and White, D., 1992. Hexagon mosaic maps for display of univariate and bivariate geographical data. Cartography and Geographic Information Systems 19(4), pp. 228-236.

Cauvin, C., Escobar, F. and Serradj, A., 2010. Thematic Cartography, New Approaches in Thematic Cartography. Vol. 3, Wiley-ISTE.

Choi, M., Shin, S., Choi, J., Langevin, S., Bethune, C., Horne, P., Kronenfeld, N., Kannan, R., Drake, B., Park, H. et al., 2018. Topicontiles: Tile-based spatio-temporal event analytics via exclusive topic modeling on social media. In: Proceedings of the 2018 CHI Conference on Human Factors in Computing Systems, pp. 1-11.

Davoine, P.-A., Mathian, H., Saint-Marc, C., Blaise, J.-Y. and Kaddouri, L., 2015. The visual representations of territorial dynamics: Retrospective and input from new computing environments. In: ICC 2015.

Delmelle, E., Dony, C., Casas, I., Jia, M. and Tang, W., 2014. Visualizing the impact of space-time uncertainties on dengue fever patterns. International Journal of Geographical Information Science 28(5), pp. 1107-1127.

Delort, J.-Y., 2010. Hierarchical cluster visualization in web mapping systems. In: Proceedings of the 19th international conference on World wide web, pp. 1241-1244.

Demšar, U., Buchin, K., van Loon, E. E. and ShamounBaranes, J., 2015. Stacked space-time densities: a geovisualisation approach to explore dynamics of space use over time. GeoInformatica 19(1), pp. 85-115.

Desjardins, M., Hohl, A. and Delmelle, E., 2020. Rapid surveillance of covid-19 in the united states using a prospective space-time scan statistic: Detecting and evaluating emerging clusters. Applied Geography 118, pp. 102202.

Dukaczewski, D., 2005. Entities-cartotropic method of selection of static and dynamic variables for temporal cartographic animations. In: Proceedings of the XXII rd ACI/ICA International Cartographic Conference.

Gatalsky, P., Andrienko, N. and Andrienko, G., 2004. Interactive analysis of event data using space-time cube. In: Proceedings. Eighth International Conference on Information Visualisation, 2004. IV 2004., IEEE, pp. 145-152.

Gautier, J., Davoine, P.-A. and Cunty, C., 2020. Geovisualization and harmonic analysis for the exploratory search of localized cyclic recurrences in spatio-temporal event data. Geomatica pp. 1-23.

Gonçalves, T., Afonso, A. P. and Martins, B., 2016. Why not both? combining $2 \mathrm{~d}$ maps and $3 \mathrm{~d}$ space-time cubes for human trajectory data visualization. In: Proceedings of the 30th International BCS Human Computer Interaction Conference: Fusion!, pp. 1-10.
Green, M., 1998. Toward a perceptual science of multidimensional data visualization: Bertin and beyond. ERGO/GERO Human Factors Science.

Hägerstraand, T. et al., 1970. What about people in regional science? Papers in Regional Science 24(1), pp. 724.

Harrower, M., Fabrikant, S. and Dodge, M., 2008. The role of map animation in geographic visualization.

Huang, G., Govoni, S., Choi, J., Hartley, D. and Wilson, J., 2008. Geovisualizing data with ring maps. ArcUser 11(1), pp. 54-55.

Kapler, T. and Wright, W., 2005. Geotime information visualization. Information Visualization 4(2), pp. 136-146. Kirby, R. S., Delmelle, E. and Eberth, J. M., 2017. Advances in spatial epidemiology and geographic information systems. Annals of Epidemiology 27(1), pp. $1-9$. GIS and Spatial Methods in Epidemiology Symposium.

Kraak, M.-J., 2008. Geovisualization and time-new opportunities for the space-time cube. Geographic visualization: concepts, tools and applications pp. 293-306.

Kraak, M.-J. and Ormeling, F., 2011. Cartography: visualization of spatial data. Guilford Press.

Lan, Y., Desjardins, M. R., Hohl, A. and Delmelle, E., 2021. Geovisualization of covid-19: State of the art and opportunities. Cartographica: The International Journal for Geographic Information and Geovisualization p. e20200027.

League, C. and Kennelly, P., 2019. Cartographic symbol design considerations for the space-time cube. The Cartographic Journal 56(2), pp. 117-133.

$\mathrm{Li}, \mathrm{X} ., 2010$. The time wave in time space: a visual exploration environment for spatio-temporal data.

Liu, Y., He, Z. and Zhou, X., 2020. Space-time variation and spatial differentiation of covid-19 confirmed cases in hubei province based on extended gwr. ISPRS International Journal of Geo-Information 9(9), pp. 536.

MacEachren, A. M., 2004. How maps work: representation, visualization, and design. Guilford Press.

Meier, S., 2016. The marker cluster. International Journal of Agricultural and Environmental Information Systems 7 , pp. $28-43$.

Müller, H. and Louwsma, M., 2021. The role of spatiotemporal information to govern the covid-19 pandemic: A european perspective. ISPRS International Journal of GeoInformation 10(3), pp. 166.

Munzner, T., 2014. Visualization analysis and design. CRC press.

Piarroux, R., 2019. Choléra. Haïti 2010-2018: histoire d'un désastre. Cnrs.

Purwanto, P., Utaya, S., Handoyo, B., Bachri, S., Astuti, I. S., Utomo, K. S. B. and Aldianto, Y. E., 2021. Spatiotemporal analysis of covid-19 spread with emerging hotspot analysis and space-time cube models in east java, indonesia. ISPRS International Journal of Geo-Information 10(3), pp. 133.

Saint-Marc, C., Davoine, P.-A. and Villanova-Oliver, M., 2014. Methods for mapping volcanic events overlaid across time. Journal of Maps 10(2), pp. 249-256.

Seebacher, D., Häuäler, J., Hundt, M., Stein, M., Müller, H., Engelke, U. and Keim, D., 2018. Visual analysis of spatio-temporal event predictions: Investigating the spread dynamics of invasive species. IEEE Transactions on Big Data.

Swedberg, B. and Peuquet, D., 2017. Perse: visual analytics for calendar related spatiotemporal periodicity detection and analysis. GeoInformatica 21(3), pp. 577-597.

Tufte, E. R., 1983. The visual display of quantitative information graphics press. Cheshire, Connecticut. n.d. 